\title{
Bioenergetic dysfunction in Huntington's disease human cybrids
}

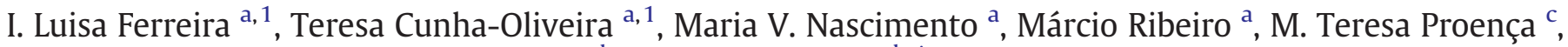 \\ Cristina Januário ${ }^{\mathrm{c}}$, Catarina R. Oliveira ${ }^{\mathrm{a}, \mathrm{b}}, \mathrm{A}$. Cristina Rego ${ }^{\mathrm{a}, \mathrm{b}, *}$ \\ a Center for Neuroscience and Cell Biology, 3004-504 Coimbra, Portugal \\ ${ }^{\mathrm{b}}$ Faculty of Medicine, University of Coimbra, 3004-504 Coimbra, Portugal \\ c Neurology Unit of Coimbra, University Hospital, 3004-504 Coimbra, Portugal
}

\section{A R T I C L E I N F O}

Article history:

Received 3 February 2011

Revised 30 April 2011

Accepted 28 May 2011

Available online 13 June 2011

Keywords:

Amino acids

Cybrids

Energy metabolism

Glycolysis

Huntington's disease

Mitochondria

Tricarboxylic acid cycle

\begin{abstract}
A B S T R A C T
In this work we studied the mitochondrial-associated metabolic pathways in Huntington's disease (HD) versus control (CTR) cybrids, a cell model in which the contribution of mitochondrial defects from patients is isolated. HD cybrids exhibited an interesting increase in ATP levels, when compared to CTR cybrids. Concomitantly, we observed increased glycolytic rate in HD cybrids, as revealed by increased lactate/pyruvate ratio, which was reverted after inhibition of glycolysis. A decrease in glucose-6-phosphate dehydrogenase activity in HD cybrids further indicated decreased rate of the pentose-phosphate pathway. ATP levels of HD cybrids were significantly decreased under glycolysis inhibition, which was accompanied by a decrease in phosphocreatine. Nevertheless, pyruvate supplementation could not recover HD cybrids' ATP or phosphocreatine levels, suggesting a dysfunction in mitochondrial use of that substrate. Oligomycin also caused a decrease in ATP levels, suggesting a partial support of ATP generation by the mitochondria. Nevertheless, mitochondrial NADH/NAD levels were decreased in $\mathrm{HD}$ cybrids, which was correlated with a decrease in pyruvate dehydrogenase activity and protein expression, suggesting decreased tricarboxylic acid cycle (TCA) input from glycolysis. Interestingly, the activity of alpha-ketoglutarate dehydrogenase, a critical enzyme complex that links the TCA to amino acid synthesis and degradation, was increased in HD cybrids. In accordance, mitochondrial levels of glutamate were increased and alanine was decreased, whereas aspartate and glutamine levels were unchanged in HD cybrids. Conversely, malate dehydrogenase activity from total cell extracts was unchanged in HD cybrids. Our results suggest that inherent dysfunction of mitochondria from HD patients affects cellular bioenergetics in an otherwise functional nuclear background.
\end{abstract}

(c) 2011 Elsevier Inc. All rights reserved.

\section{Introduction}

Huntington's disease (HD) is an autosomal dominant neurodegenerative disease, clinically characterized by psychiatric disturbances, progressive cognitive impairment and choreiform movements. These symptoms are associated with the selective atrophy and neuronal loss in the striatum and cortex. The disease is caused by a mutation at the $5^{\prime}$ terminal of the huntingtin gene, involving the expansion of CAG triplet, which encodes for glutamine. Mutant huntingtin may be cleaved by proteases originating neurotoxic fragments, and also undergoes conformational changes that lead to the formation of protein aggregates (Gil and Rego, 2008, for review).

\footnotetext{
* Corresponding author at: Center for Neuroscience and Cell Biology, and Faculty of Medicine, University of Coimbra (polo I), Rua Larga, 3004-504 Coimbra, Portugal. Fax: + 351239822776 .

E-mail address: a.cristina.rego@gmail.com (A.C. Rego).

1 The authors contributed equally to this manuscript.
}

Among several mechanisms of neurodegeneration, mutant huntingtin is related to mitochondrial dysfunction and relevant changes in energy metabolism, in both central and peripheral cells which may underlie cell death (Gil and Rego, 2008). HD patients present high levels of lactate in both the striatum (Jenkins et al., 1993) and cortex (Jenkins et al., 1993; Koroshetz et al., 1997), and increased lactate/pyruvate ratio in the cerebrospinal fluid (CSF) (Koroshetz et al., 1997). Striatal glucose metabolism was also shown to be decreased, whereas oxygen metabolism is preserved in early symptomatic HD patients (Powers et al., 2007b). Moreover, increased glucose metabolism and ATP levels were found in brain tissue of HD N171-82Q mice, suggesting that the neuronal damage in HD tissue may be associated with increased energy metabolism at the tissue level, leading to modified levels of various intermediary metabolites (Olah et al., 2008). A decrease in mitochondrial complexes II-III activity and a decrease in succinate oxidation were also found in striatal tissue from HD patients (Schapira, 1998; Tabrizi et al., 1999). Accordingly, the expression of two subunits of the complex II is decreased in the striatum of HD patients, affecting the dehydrogenase activity of the complex (Benchoua et al., 2006). A small decrease in complex IV in brain samples was also reported (Browne et al., 1997; Gu et al., 1996; Schapira, 1998; Tabrizi et al., 1999). 
In peripheral tissues, mutant huntingtin was reported to affect the activity of mitochondrial complex I (Arenas et al., 1998) and complexes II-III (Ciammola et al., 2006; Turner et al., 2007) along with mitochondrial depolarization, cytochrome c release and increased caspases activity (Ciammola et al., 2006; Turner et al., 2007) in skeletal muscle. In platelets from HD patients, some authors found a decrease in complex I activity (Parker et al., 1990), whereas others reported no changes in the activity of mitochondrial complexes $(\mathrm{Gu}$ et al., 1996; Powers et al., 2007a). Moreover, the phosphocreatine/ inorganic phosphate ratio was significantly decreased in resting muscle (Koroshetz et al., 1997) of HD patients, evidencing bioenergetic changes in HD peripheral tissues.

These data suggest the involvement of mitochondrial defects in HD pathogenesis. Mutant huntingtin may cause mitochondrial dysfunction by either perturbing transcription of nuclear-encoded mitochondrial proteins or by directly interacting with the organelle, thus evoking defects in mitochondrial dynamics, organelle trafficking and fission and fusion, which, in turn, may result in bioenergetic failure in HD (Bossy-Wetzel et al., 2008). In fact, mutant huntingtin was previously shown to interact with neuronal mitochondria of YAC72 transgenic mice (Panov et al., 2002), suggesting that mitochondrial calcium abnormalities associated with HD pathogenesis may be due to a direct effect of mutant huntingtin on the organelle (Choo et al., 2004; Panov et al., 2002).

Moreover, mutant huntingtin fragments can directly induce the opening of the mitochondrial permeability transition pore in isolated mouse liver mitochondria, with the consequent release of cytochrome c, as well as a decrease in the calcium threshold necessary to induce it (Choo et al., 2004), favoring the hypothesis that mutant huntingtin interacting with mitochondria may well lead to mitochondrial modifications independently of damage on mtDNA.

Mitochondria from lymphoblasts of HD patients have been shown to present increased susceptibility to apoptotic stimuli due to an abnormal mitochondrial transmembrane potential (Sawa et al., 1999). Lymphoblasts derived from HD patients also showed increased stress-induced apoptotic cell death associated with caspase- 3 activation, abnormal calcium homeostasis and mitochondrial dysfunction (Bezprozvanny and Hayden, 2004; Panov et al., 2002; Sawa et al., 1999). Previously, we demonstrated that HD human peripheral blood cells, particularly B lymphocytes, are endowed with increased expression of Bax and decreased mitochondrial membrane potential (Almeida et al., 2008), further suggesting that an adverse effect of mutant huntingtin is not limited to neurons.

In addition, we previously found that HD cybrids, obtained from the fusion of HD platelets with NT2 rho0 cells, depleted of mitochondrial DNA, do not exhibit significant modifications in the activity of mitochondrial respiratory chain complexes I-IV or in mtDNA sequence variations, suggestive of a primary role in mitochondrial susceptibility in the subpopulation of HD carriers studied (Ferreira et al., 2010). In accordance, Swerdlow et al. (1999) showed that HD cybrids did not present changes in mitochondrial respiratory chain activity, oxidative stress or calcium homeostasis. Conversely, increased mitochondrial Bim and Bak levels, and a slight release of cytochrome $\mathrm{c}$ in untreated HD cybrids explained their moderate susceptibility to mitochondrial-dependent apoptosis (Ferreira et al., 2010). These changes were translated into increased susceptibility of HD cybrids to undergo mitochondrial-dependent apoptosis when subjected to complex II inhibition with 3-nitropropionic acid (3-NP) or to apoptosis elicited by the classic inducer staurosporine (Ferreira et al., 2010). Despite unchanged activity of mitochondrial complexes, this cell model presented evidences of mitochondrial dysfunction based on significant changes on mitochondrial membrane potential and increased ROS generation. Taking into account our previous data and the importance of metabolic defects in HD pathogenesis, the aim of this study was to investigate changes in energy metabolism in HD human cybrid lines.

\section{Materials and methods}

\section{Materials}

Opti-MEM was purchased from GIBCO (Paisley, UK). RPMI-1640, protease inhibitor cocktail (chymostatin, pepstatin A, leupeptin and antipain), 3-NP and penicillin/streptomycin, $\beta$-Nicotinamide adenine dinucleotide hydrate (NAD), thiamine pyrophosphate (TPP), $\mathrm{CoASH}$, sodium pyruvate, malate, citrate synthase from porcine heart, acetylCoA, $\beta$-Nicotinamide adenine dinucleotide phosphate hydrate (NADP), glucose 6-phosphate and maleimide were from Sigma Chemical Co. (St Louis, MO, USA). All other reagents were of analytical grade.

\section{Cybrid production, culture and incubation with 3-NP}

Cybrids (cytoplasmic hybrid systems) were produced after fusion of mitochondrial DNA-depleted human teratocarcinoma cells (rho0 NT2 cells, obtained from Dr. R. H. Swerdlow, University of Virginia, Charlottesville, VA, USA), with human platelets isolated from six genetically and clinically confirmed HD patients (42-44 CAG repeats) from pre-identified Portuguese families and three age-matched healthy controls (without any neurological disease) as described in Ferreira et al. (2010). Cybrids were cultured in Opti-MEM medium supplemented with $10 \%$ fetal calf serum, penicillin $(100 \mathrm{U} / \mathrm{ml})$, streptomycin $(100 \mu \mathrm{g} / \mathrm{ml})$ and maintained at $37^{\circ} \mathrm{C}$ in humidified incubator containing 95\% air and 5\% $\mathrm{CO}_{2}$. Experiments were performed with cybrids less than 2 months in culture, as previously described by us (Ferreira et al., 2010). In experiments aiming to inhibit glycolysis, the commonly used culture medium (Opti-MEM) was replaced by RPMI medium with (+ Gluc) or without glucose. In some experiments, $2 \mu \mathrm{g} / \mathrm{ml}$ oligomycin was added to glucosecontaining or glucose-free medium in order to inhibit mitochondrial ATP synthesis. In another set of experiments, RPMI without glucose was supplemented with $2 \mathrm{mM}$ 2-deoxy-D-glucose (+2-DOG), a competitive inhibitor of glucose metabolism (Wick et al., 1957), and cells were maintained in these media for $8 \mathrm{~h}$ prior to the experiments. To further analyze the importance the glycolytic metabolism in cell bioenergetic status, in some experiments RPMI medium without glucose plus 2-DOG was supplemented with $10 \mathrm{mM}$ pyruvate (2-DOG + Pyr) to directly feed the mitochondria.

Cybrids were plated on multiwell chambers or flasks at a density of $0.06 \times 10^{6}$ cells $/ \mathrm{cm}^{2}$, one day before the experiments, in order to allow the desired confluence. Where indicated, cells were incubated in culture medium in the absence or presence of $3-\mathrm{NP}(0.1,1$ or $10 \mathrm{mM})$ for $24 \mathrm{~h}$ or in the absence or presence of metabolic inhibitors, for $8 \mathrm{~h}$, as described in figure legends.

\section{Measurement of ATP and phosphocreatine}

Cells were washed with ice-cold PBS, scraped, and extracted with $0.6 \mathrm{M}$ perchloric acid, supplemented with $25 \mathrm{mM}^{\mathrm{EDTA}-\mathrm{Na}^{+} \text {, and }}$ centrifuged at $20,800 \mathrm{~g}$ for $5 \mathrm{~min}$ at $4{ }^{\circ} \mathrm{C}$. The resulting pellet was solubilized with $1 \mathrm{M} \mathrm{NaOH}$ and further analyzed for total protein content by the Bio-Rad Protein assay. After neutralization with $3 \mathrm{M}$ $\mathrm{KOH} / 1.5 \mathrm{M}$ Tris, samples were centrifuged at 20,800 $\mathrm{g}$ for $5 \mathrm{~min}$ at $4{ }^{\circ} \mathrm{C}$. The resulting supernatants were assayed for ATP determination by separation in a reverse-phase high-performance liquid chromatography (HPLC), with detection at $254 \mathrm{~nm}$, as described previously (Stocchi et al., 1985). ADP and AMP levels were undetectable in our samples. Peak identity was determined by the retention time compared with standards and the amount of ATP was determined by using a concentration standard curve. These samples were also used to determine intracellular phosphocreatine levels, by following $\mathrm{NADP}^{+}$reduction at $340 \mathrm{~nm}$, mediated by ATP production by creatine kinase, in the presence of hexokinase and glucose-6-phosphate 
dehydrogenase (G6PD), by using a Microplate Spectrophotometer SpectraMax Plus 384 (Molecular Devices, USA), according to a previously described method (Heinz and Weißer, 1985). The results were expressed in pmol phosphocreatine or ATP per mg protein and converted in percentage of controls.

\section{Assessment of intracellular pyruvate and lactate}

Cells were washed with ice-cold PBS and extracted with $0.6 \mathrm{M}$ perchloric acid supplemented with 25 mM EDTA-Na ${ }^{+}$. Cell extracts were centrifuged at $20,800 \mathrm{~g}$ for $5 \mathrm{~min}$ at $4{ }^{\circ} \mathrm{C}$ in order to remove cell debris. Lactate and pyruvate were determined in the supernatant by using kits from Randox Laboratories (Antrim, U.K.) and Ben SRL (Milan, Italy), respectively. Results of lactate and pyruvate content were obtained in $\mathrm{pmol} / \mathrm{mg}$ protein and expressed as a ratio of lactate/pyruvate.

\section{Quantification of mitochondrial NADH/NADt ratio}

Cells were washed in ice-cold PBS, extracted in sucrose buffer and then homogenized by using a Thomas USA 0872 potter, at $4{ }^{\circ} \mathrm{C}$. Extracts were then centrifuged at $560 \mathrm{~g}$ in order to remove nuclear debris and the resulting supernatant was centrifuged at $12,000 \mathrm{~g}$ for $20 \mathrm{~min}$ at $4{ }^{\circ} \mathrm{C}$ in order to obtain mitochondrial sediment. Mitochondrial fraction was ressuspended in a medium containing $20 \mathrm{mM} \mathrm{NaHCO}, 100 \mathrm{mM}$ $\mathrm{Na}_{2} \mathrm{CO}_{3}, 10 \mathrm{mM}$ nicotinamide and $0.05 \%$ Triton $\mathrm{X}-100$ at $4{ }^{\circ} \mathrm{C}$ and centrifuged at $15,300 \mathrm{~g}$ for $5 \mathrm{~min}$ at $4{ }^{\circ} \mathrm{C}$. An aliquot of the supernatant was then incubated at $60{ }^{\circ} \mathrm{C}$ for $30 \mathrm{~min}$ in order to selectively decompose the thermo sensible $\mathrm{NAD}^{+}$. The remaining supernatant was kept on ice until the determination of total NAD $\left(N A D_{t}\right)$. Aliquots of $50 \mu \mathrm{l}$ were then incubated with $840 \mu \mathrm{l}$ reaction buffer containing: $100 \mathrm{mM}$ Tris, pH 7.8; 5 mM EDTA, 0.5 mM 3-(4,5-dimethylthiazol-2yl)-2,5-diphenyltetrazolium bromide (MTT), $0.2 \mathrm{mg} / \mathrm{ml}$ alcohol dehydrogenase and $10 \mu \mathrm{l}$-ethyldibenzopyrazine ethyl sulfate salt (PES) $200 \mathrm{mM}$ for $5 \mathrm{~min}$ at $25^{\circ} \mathrm{C}$, and then $100 \mu \mathrm{l}$ of $6 \mathrm{M}$ ethanol was added to each sample. Extracts were centrifuged at $15,300 \mathrm{~g}$ for $30 \mathrm{~s}$ at $25^{\circ} \mathrm{C}$ and quantified by using a colorimetric assay based on MTT reduction that occurs at $570 \mathrm{~nm}$ during 2 min (Umemura and Kimura, 2005) by using a microplate reader Spectra Max Plus 384 (Molecular Devices, USA). Protein was quantified by BioRad protein assay (Bradford method) and results normalized as $\mathrm{pmol} / \mathrm{mg}$ protein, and presented as NADH/NADt ratio.

\section{Tricarboxylic acid cycle (TCA) enzymatic activities}

Pyruvate, alpha-ketoglutarate and malate dehydrogenases activities were measured as described previously (Nulton-Persson and Szweda, 2001; Starkov et al., 2004; Zhou et al., 2008). Total cell

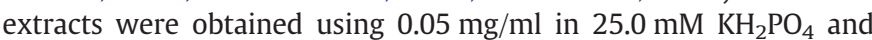
0.5 mM EDTA, pH 7.25, containing $0.01 \%$ Triton X-100 and protein quantified using BioRad protein assay. Enzyme activities were determined by following the rate of NADH formation at $340 \mathrm{~nm}$ by using a microplate reader Spectra Max Plus 384 (Molecular Devices, USA). For pyruvate dehydrogenase (PDH), $15 \mu$ g of cell sample were mixed with $2.5 \mu \mathrm{M}$ rotenone, $0.5 \mathrm{mM}$ of $\mathrm{NAD}^{+}, 0.2 \mathrm{mM}$ thiamine pyrophosphate $\left(\mathrm{TPP}^{+}\right.$), $0.04 \mathrm{mM} \mathrm{CoASH}$, and $4 \mathrm{mM}$ pyruvate (Zhou et al., 2008). For alpha-ketoglutarate dehydrogenase (KGDH) activity, $15 \mu \mathrm{g}$ of cell sample were mixed with $0.3 \mathrm{mM} \mathrm{TPP}^{+}, 10 \mathrm{mM} \mathrm{CaCl}_{2}$, $0.2 \mathrm{mM} \mathrm{MgCl}$, $5 \mathrm{mM}$ alpha-ketoglutarate (KG), $1 \mu \mathrm{M}$ rotenone, $0.2 \mathrm{mM} \mathrm{NAD}^{+}$, and $0.14 \mathrm{mM}$ CoASH (Starkov et al., 2004). For malate dehydrogenase activity, $15 \mu \mathrm{g}$ of protein were used and mixed with $40 \mu \mathrm{M}$ rotenone, $5 \mathrm{mM} \mathrm{MgCl} 2,25 \mathrm{mM}$ malate, $1 \mathrm{unit} / \mathrm{ml}$ of citrate synthase, $0.3 \mathrm{mM}$ acetyl-CoA, and $10 \mathrm{mM} \mathrm{NAD}^{+}$(Nulton-Persson and Szweda, 2001). The activities were calculated as the absorbance units per min and per mg protein.

\section{Measurement of G6PD activity}

G6PD activity was determined according to Choo et al. (2005), with some minor modifications. Briefly, cell samples $(30 \mu \mathrm{g})$ were transferred to a 96 -well plate containing $150 \mu \mathrm{L}$ of reaction buffer containing: $0.38 \mathrm{mM} \mathrm{NADP}{ }^{+}, 6.3 \mathrm{mM} \mathrm{MgCl}_{2}, 3.3 \mathrm{mM}$ glucose-6phosphate, and $5 \mathrm{mM}$ maleimide (to inhibit 6-phosphogluconate dehydrogenase -6PGD- activity) in $50 \mathrm{mM}$ Tris- $\mathrm{HCl}(\mathrm{pH} 7.5)$ and the total volume of $200 \mu \mathrm{L}$ was adjusted with water. NADPH production was continuously monitored at $340 \mathrm{~nm}$, using a microplate reader Spectra Max Plus 384 (Molecular Devices, USA) at $37^{\circ} \mathrm{C}$, and the activity was calculated in absorbance units per min per mg protein.

Determination of pyuvate dehydrogenase (PDH) E1 $\alpha$ subunit protein levels and phosphorylation

PDH expression and phosphorylation were assessed using a kit from MitoSciences (Oregon, USA) following the manufacturer instructions and using the solutions provided in the kit. Briefly, cells were plated at confluent density, allowed to adhere, and fixed with $3.7 \%$ formaldehyde $/ 4 \%$ sucrose. Cells were permeabilized, blocked and incubated with total PDH E1 $\alpha$ combined with another antibody against the phosphorylated forms of PDH E1 $\alpha$ at pSer 232, pSer293 or pSer 300, and developed by dual colorimetric detection. Cell density was normalized after Janus Green staining. Absorbance was monitored using a microplate reader Spectra Max Plus 384 (Molecular Devices, USA).

Assessment of mitochondrial levels of aspartate, glutamate, glutamine and alanine by HPLC

The levels of the amino acids aspartate, glutamate, glutamine and alanine were measured in mitochondrial fractions obtained from CTR and HD cybrids, prepared as described for the analysis of mitochondrial $\mathrm{NADH} / \mathrm{NAD}_{\mathrm{t}}$ ratio. Mitochondrial pellets were resuspended in $1 \mathrm{M} \mathrm{NaOH}$.

The amino acids were analyzed in a Gilson-ASTED HPLC system. Samples were separated on a Hichrom ACE type column $(150 \times 4.6 \mathrm{~mm}$, $5 \mu \mathrm{M} \mathrm{C18}$ ) at a flow rate of $2.5 \mathrm{ml} / \mathrm{min}$ for $45 \mathrm{~min}$, using a ternary solvent system consisting of solvent A [37.5 mM sodium phosphate, $50 \mathrm{mM}$ propionic acid, $7 \%$ acetonitrile, and 3\% dimethyl sulfoxide ( $\mathrm{pH} 6.2$ )], solvent B (40\% acetonitrile, 33\% methanol, and 7\% dimethyl sulfoxide), and solvent $\mathrm{C}$ [ $62.5 \mathrm{mM}$ sodium phosphate, $50 \mathrm{mM}$ propionic acid, $7 \%$ acetonitrile, and $3 \%$ dimethyl sulfoxide ( $\mathrm{pH}$ 5.5)]. The amino acids were detected as fluorescent derivatives after precolumn derivatization with o-phthaldialdehyde/2-mercaptoethanol (OPA/MCE), using a Gilson fluorescent detector model 121, with excitation at $340 \mathrm{~nm}$ and emission at $410 \mathrm{~nm}$. Amino acid concentration was determined by comparison with peak areas of amino acid standards, and expressed in nanomol per $\mathrm{mg}$ of protein. Protein levels were determined by the BioRad protein assay.

\section{Statistical analysis}

No significant differences in biochemical studies were observed between the six HD cybrid lines and between the three CTR cybrids used in this work. Therefore data were expressed as the mean \pm S.E.M. of the number of experiments indicated in the figure legends. Comparisons between multiple groups were performed with a twoway analysis of variance (ANOVA), followed by Bonferroni post-test for comparison between experimental groups. Student's $t$ test was also performed for comparison between two Gaussian populations, as described in figure legends. Significance was accepted at $\mathrm{p}<0.05$. 


\section{Results}

\section{Glycolysis is stimulated in HD cybrids}

We initially characterized the energy metabolism in HD cybrid lines derived from symptomatic HD patients. Intracellular ATP levels were analyzed in order to evaluate the bioenergetic status of HD versus CTR cybrids in the absence or in the presence of 3-NP (Fig. 1). In both basal and in $0.1 \mathrm{mM}$ 3-NP-treated conditions, HD cybrids presented a significant increase in total ATP levels when compared with CTR cybrids. Although still being higher in HD cybrids compared to CTR cybrids, ATP levels slightly decreased in the presence of 1 and $10 \mathrm{mM}$ 3-NP (Fig. 1); no measurable levels of ADP or AMP were detected in these conditions.

Since we observed an increase in total ATP levels in HD cybrids, the glycolytic metabolic rate in HD versus CTR cybrids was then compared before and after glycolysis inhibition, for $8 \mathrm{~h}$ (Fig. 2). For this purpose, the usual cell culture medium Opti-MEM (only commercialized containing glucose) was changed to RPMI-1640 medium, in order to allow testing experimental conditions lacking glucose. No differences were observed in lactate/pyruvate ratio, under basal conditions, in both cell lines cultured in Opti-MEM or RPMI+Gluc (data not shown). Interestingly, HD cybrids exhibited increased lactate/pyruvate ratio compared to CTR cybrids (Fig. 2A), suggesting an increased glycolytic rate, which may underlie increased ATP production in HD cybrids. Indeed, the difference in lactate/pyruvate ratio in HD and CTR cybrids was abolished upon glycolysis inhibition, achieved by adding 2-DOG to glucose-free medium (Fig. 2A), further suggesting a higher role for glycolysis in HD cybrid bioenergetics. Indeed, lactate/pyruvate ratio was decreased in CTR cybrids in response to glycolysis inhibition, and largely decreased in HD cybrids (Fig. 2A). HD cells also showed a decreased activity of G6PD, a key enzyme of the pentose phosphate pathway (Fig. 2B), suggesting that in HD cybrids glucose is preferentially metabolized through the glycolytic pathway.

Partial dysfunction in mitochondrial bioenergetics underlies the stimulation of glycolytic pathway in HD cybrids

In order to determine the importance of the glycolytic pathway for ATP production in these cells, we further examined the changes in ATP levels in CTR and HD cybrids before and after glycolysis or ATP synthase inhibition. Glucose-free medium supplemented with 2-DOG, previously shown to inhibit glycolysis (Fig. 2), largely and significantly decreased the intracellular levels of ATP in HD cybrids, in contrast with CTR cybrids (Fig. 3A). Inhibition of ATP synthase with oligomycin in the presence of glucose decreased ATP production in

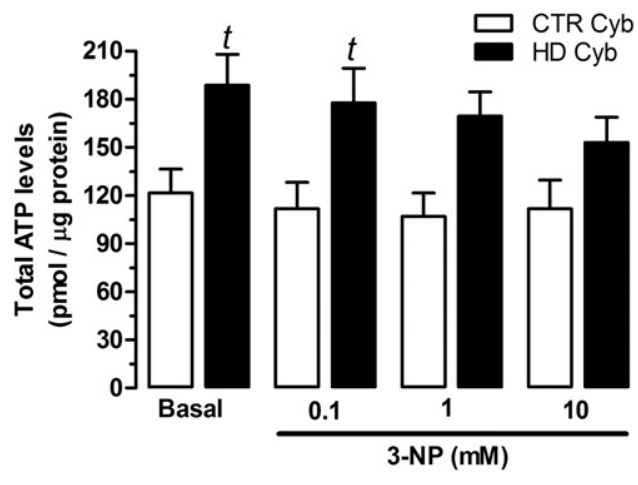

Fig. 1. Endogenous ATP levels in control (CTR) and HD human cybrids under basal conditions and after exposure to 3-NP. Cells were incubated in the absence (basal) or in the presence of $0.1,1$ and $10 \mathrm{mM}$ 3-NP for $24 \mathrm{~h}$. Intracellular ATP was determined by HPLC as described in Materials and methods. Data are expressed as the mean \pm S.E.M. from 6 to 8 independent experiments performed in duplicates. Statistical analysis was performed by $t$ test; $t \mathrm{P}<0.05$ when compared to the respective CTR Cyb.
A
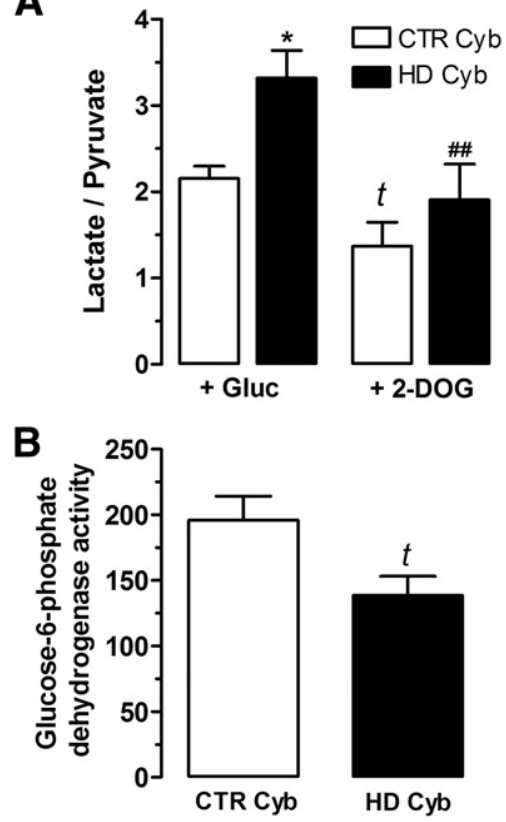

Fig. 2. Glycolysis and pentose-phosphate pathways in control (CTR) and HD cybrids. A) Intracellular lactate/pyruvate ratio levels. Cells were incubated for $8 \mathrm{~h}$ in glucosecontaining medium (RPMI + Gluc) or upon glycolysis inhibition achieved in the absence of added glucose and in the presence of $2 \mathrm{mM}$ 2-deoxyglucose (+2-DOG). B) Activity of glucose-6-phosphate dehydrogenase, a key enzyme of the pentose phosphate pathway. Cell samples obtained from CTR and HD cybrids were used to evaluate the reduction of $\mathrm{NADP}^{+}$by G6PD. Data are expressed as the mean \pm S.E.M. from 4 to 8 independent experiments performed in duplicate or triplicate. Statistical analysis was performed by Student's $t$ test; $t \mathrm{P}<0.05$, when compared to CTR Cyb ( + Gluc) or by two-way ANOVA followed by Bonferroni post test $* \mathrm{P}<0.05$ when compared to CTR Cyb (+ Gluc); ${ }^{\# \# ~} \mathrm{P}<0.01$ when compared to the HD Cyb (+ Gluc).

HD, compared to CTR cybrids, and no difference was observed between the two cell lines (Fig. 3A). In the absence of glucose, cells can use exogenous pyruvate (or lactate) to generate ATP by oxidative phosphorylation (Budd and Nicholls, 1996; Rego et al., 2001b). Interestingly, we observed that metabolically feeding mitochondria with pyruvate under conditions of glycolysis inhibition did not recover ATP levels in HD cybrids (Fig. 3A). Inhibition of ATP synthase in the absence of active glycolysis drastically decreased ATP levels in both HD and CTR cybrids. Slight changes in ATP levels caused by 2DOG (in glucose-free medium) and pyruvate, were observed in CTR cybrids.

We further examined the contribution of phosphocreatine, which constitutes a reservoir for rapid ATP regeneration, in HD and CTR cybrids (Fig. 3B). Upon glycolysis inhibition, phosphocreatine levels significantly decreased in HD cybrids, whereas a moderate decrease was observed in CTR cybrids, suggesting that phosphocreatine contributes to maintain the levels of ATP in HD cybrids subjected to glycolytic inhibition (Fig. 3B). Since glycolysis activation may occur in cells exhibiting decreased mitochondrial metabolic efficiency, we determined phosphocreatine levels after supplying mitochondria with pyruvate. In these conditions, phosphocreatine levels were also decreased in HD cybrids, in contrast with CTR cybrids, further suggesting an important role for phosphocreatine in maintaining cellular ATP levels (Fig. 3B). These data reveal the cellular interdependence of these two rapid generating energy metabolic pathways. These observations not only suggest that HD cybrids have increased glucose oxidation rate, but also that this may be a compensatory response to maintain cellular ATP levels. However, inhibition of ATP synthase with oligomycin did not affect phosphocreatine levels either in the presence or in the absence of glycolysis, in CTR or HD cybrids. The inability of pyruvate to restore ATP or 

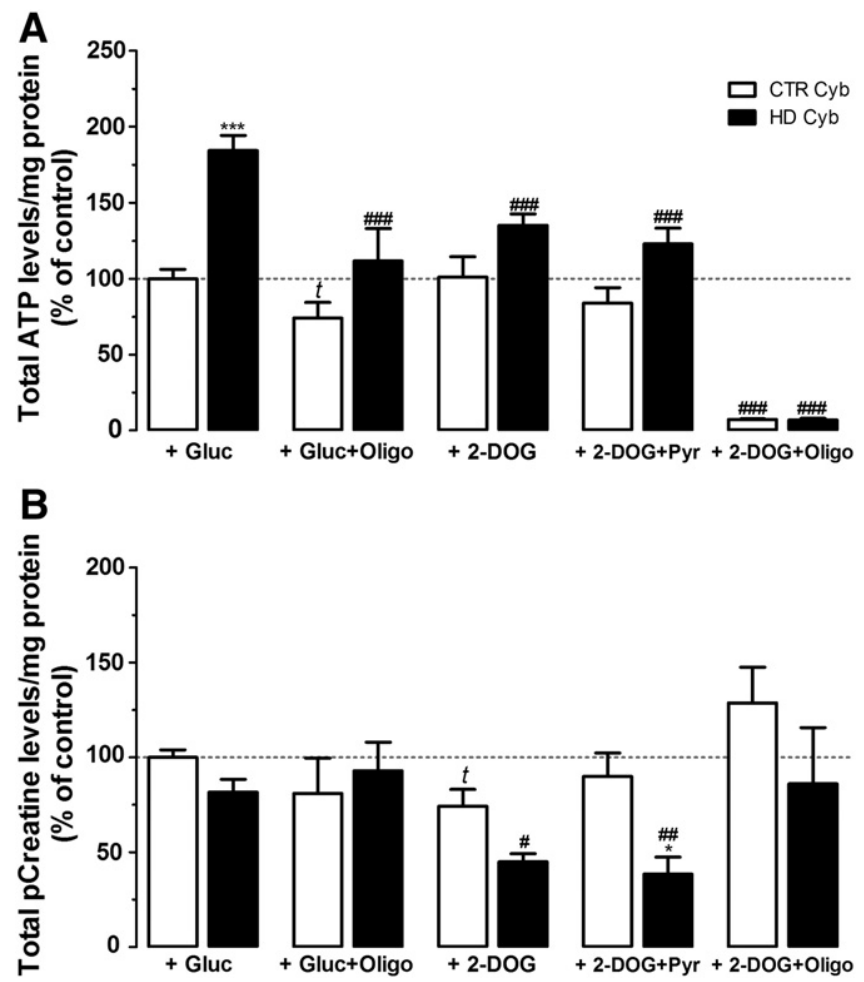

Fig. 3. Analysis of total intracellular ATP and phosphocreatine levels in HD and CTR cybrids before and after glycolysis or ATP synthase inhibition and pyruvate supplementation. Cells were incubated in the presence (+Gluc) or in the absence (plus 2-deoxyglucose, +2-DOG) of glucose in order to inhibit glycolysis, with or without oligomycin (+Gluc + Oligo or +2-DOG + Oligo) in order to inhibit ATP synthase. Pyruvate was added to directly 'feed' the mitochondria (RPMI + 2-DOG + Pyr). Data are expressed as the mean \pm S.E.M. from 4 to 6 independent experiments performed in duplicate. Statistical analysis was performed by two-way ANOVA, followed by Bonferroni post test. ${ }^{*} \mathrm{P}<0.05$, ${ }^{* * *} \mathrm{P}<0.01$ when compared to the respective CTR Cyb; \# $\mathrm{P}<0.05,{ }^{\# \#} \mathrm{P}<0.01$, \#\# $\mathrm{P}<0.001$ when compared to the appropriate basal (+ Gluc) condition.

phosphocreatine levels suggest decreased mitochondrial metabolic efficiency in HD cybrids.

Mitochondrial bioenergetics in HD cybrids is impaired due to $\mathrm{PDH}$ dysfunction

Taking into account that the activity of mitochondrial electron transport chain (ETC) complexes is maintained in HD cybrids (Ferreira et al., 2010) and that increased rate of glycolytic activity appears to exceed the capacity for oxidative metabolism in these cells, we hypothesized that HD cybrids had compromised mitochondrial

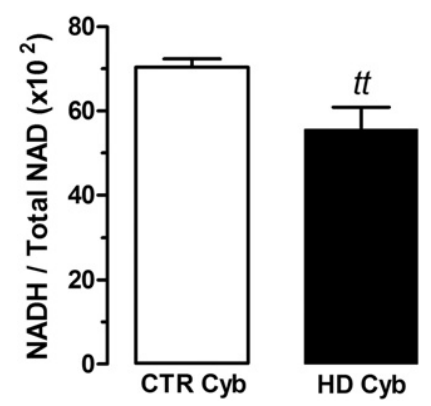

Fig. 4. Mitochondrial NADH levels. Quantification of $N A D H / N A D_{t}$ ratio was performed in mitochondrial extracts from HD Cyb or CTR Cyb. Data are expressed as the mean \pm S.E.M. from 4 to 6 independent experiments performed in duplicate. Statistical analysis was performed by Student's $t$ test. ${ }^{t t} \mathrm{P}<0.01$, when compared to CTR Cyb. function at the level of dehydrogenase enzymes associated with the TCA cycle. Thus, we measured $\mathrm{NADH} / \mathrm{NAD}_{\mathrm{t}}$ ratio in mitochondrial extracts from HD and CTR cybrids (Fig. 5). Our results show a significant decrease in mitochondrial $\mathrm{NADH} / \mathrm{NAD}_{\mathrm{t}}$ levels in $\mathrm{HD}$ cybrids, suggesting a dysfunction in mitochondrial dehydrogenases. Therefore, we determined the activity of PDH, KGDH (two highly regulated irreversible enzymes) and malate dehydrogenase (Fig. 5), which are responsible for producing a large proportion of mitochondrial NADH for complex I usage at mitochondrial ETC. In accordance with the inefficiency of pyruvate to restore ATP levels in HD cybrids, resulting in the stimulation of glycolysis in these cells, we observed a significant decrease in the PDH activity (Fig. 5A), which may explain the decrease in $\mathrm{NADH} / \mathrm{NAD}_{\mathrm{t}}$ (Fig. 4). Importantly, the decrease in PDH activity in HD cybrids was accompanied by a decrease in PDH E1 $\alpha$ subunit protein expression levels (Fig. 6A). Phosphorylation of E1 $\alpha$ subunit in HD cybrids may also contribute for a decrease in PDH activity, since a tendency for an increase in pSer232 and pSer293 PDH E1 $\alpha$ subunit was observed in these cybrids (Fig. 6B).

Nevertheless, the activity of KGDH was increased in HD versus CTR cybrids (Fig. 5B), suggesting a compensatory mechanism to counterbalance the decrease in NADH production through the PDH. However, malate dehydrogenase activity was similar in HD and CTR cybrids,

\section{A}

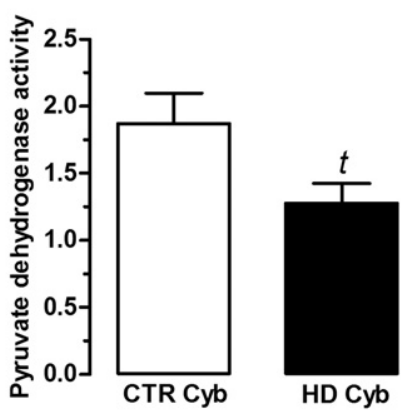

B
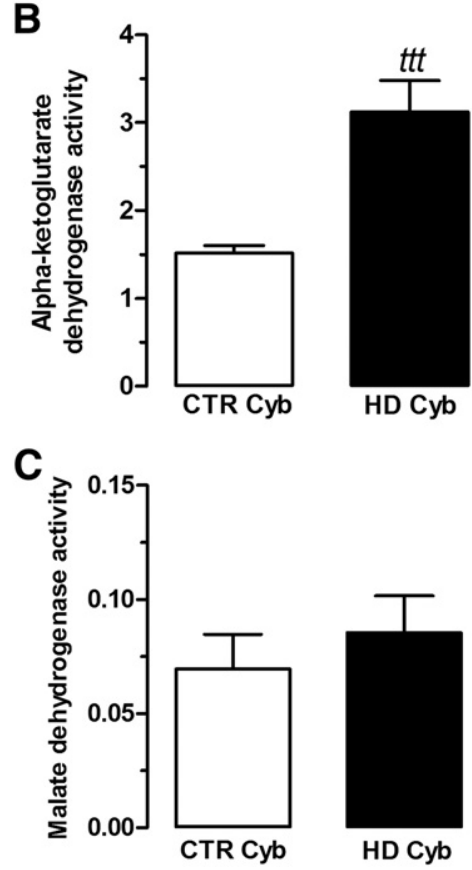

Fig. 5. Activity of dehydrogenases of the mitochondrial matrix. Total extracts were prepared from HD or CTR cybrids, and analyzed for the enzymatic activity of pyruvate dehydrogenase (PDH) (A), alpha-ketoglutarate dehydrogenase (KGDH) (B) and malate dehydrogenase $(C)$. Data are expressed as the mean \pm S.E.M. from 4 to 6 independent experiments performed in duplicates. Statistical analysis was performed by Student's $t$ test. ${ }^{t} \mathrm{P}<0.05$, ${ }^{t t t} \mathrm{P}<0.001$, when compared to CTR Cyb. 


\section{A}
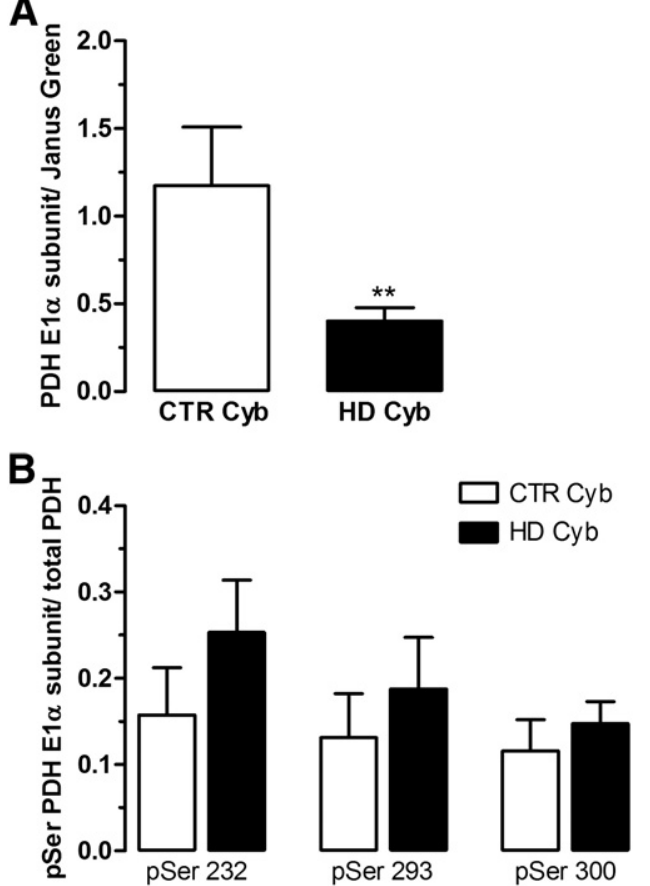

Fig. 6. Pyruvate dehydrogenase (PDH) E1 $\alpha$ subunit expression and phosphorylation. The protein expression levels and the phosphorylation of PDH subunit E1 $\alpha$ were assessed using a kit, as described in Materials and Methods. Total PDH E1 $\alpha$ expression (A) and phosphorylated forms of PDH E1 $\alpha$ at pSer 232, pSer293 or pSer 300 (B) were analyzed in CTR or HD cybrids. Statistical analysis was performed by Student's $t$ test; ${ }^{t t} \mathrm{P}<0.01$ compared with CTR cybrids. Data are the mean \pm S.E.M of total PDH E1 $\alpha /$ Janus Green or phosphorylated form/total PDH E1 $\alpha$.

measured in total cellular extracts (Fig. 5C). These results suggest that anaplerotic reactions are taking place at the level of KGDH. One of these reactions is catalyzed by glutamate dehydrogenase, which converts glutamate into $\mathrm{KG}$ and generates $\mathrm{NH}_{4}^{+}$. Thus, we next examined the mitochondrial levels of aspartate and glutamate (Fig. 7). The results indicate that although aspartate mitochondrial levels were similar in HD and CTR cybrids, mitochondria of HD cybrids presented higher levels of glutamate (Fig. 7), which may underlie the increase in KGDH activity (Fig. 5B). Furthermore, no changes in glutamine were observed in HD versus CTR cybrids (Fig. 7), excluding possible conversion of glutamine-glutamate by mitochondrial glutaminase; indeed, this enzyme may balance with the activity of glutamine

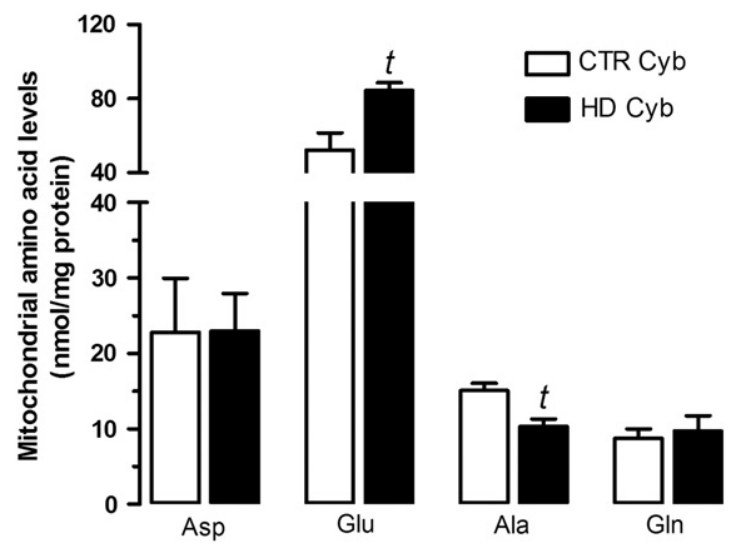

Fig. 7. Mitochondrial amino acid levels. The levels of alanine, aspartate, glutamate and glutamine were measured by HPLC in mitochondrial extracts from CTR or HD cybrids, as described in Materials and Methods. Statistical analysis was performed by Student's $t$ test; ${ }^{t} \mathrm{P}<0.05$ compared with CTR cybrids. Data are the mean \pm S.E.M. of 6-7 independent experiments. synthase, which detoxifies $\mathrm{NH}_{4}^{+}$. Conversely, mitochondrial levels of alanine were decreased, suggesting increased glutamate generation through the activity of alanine transaminase, which was previously described to be present in the mitochondrial matrix (DeRosa and Swick, 1975), apart from its most frequently reported cytosolic subcellular location. The results are summarized in Fig. 8.

\section{Discussion}

In this work we show that mitochondrial bioenergetics of HD cybrids, an ex-vivo human peripheral cell model in which the contribution of mitochondrial defects from HD patients is isolated, is compromised when compared with CTR cybrids.

Mitochondrial dysfunction is considered a common feature in the pathogenesis of neurodegenerative disorders like HD (Kim et al., 2010; Oliveira, 2010; Parker et al., 1990). Mitochondrial dysfunction constitutes a cellular hallmark for neurodegeneration and occurs as a consequence of defective mitochondrial composition, trafficking to synapses, calcium handling, ATP production, transcription abnormalities and/or ETC impairment (Rosenstock et al., 2010). Overexpression of mutant huntingtin, but not the normal protein, increases oxidative stress-induced mitochondrial fragmentation in HeLa cells, which correlates with increased caspase- 3 activation and cell death (Wang et al., 2009). Moreover, both mitochondrial loss and altered mitochondrial morphogenesis with increased mitochondrial fission and reduced fusion have been described in moderate-to-severe grade HD patients' striatal neurons (Kim et al., 2010). Furthermore, overexpression of proteins that stimulate mitochondrial fusion attenuates the toxicity of Htt proteins containing expanded polyglutamine tracts in both cells and animals (Wang et al., 2009).

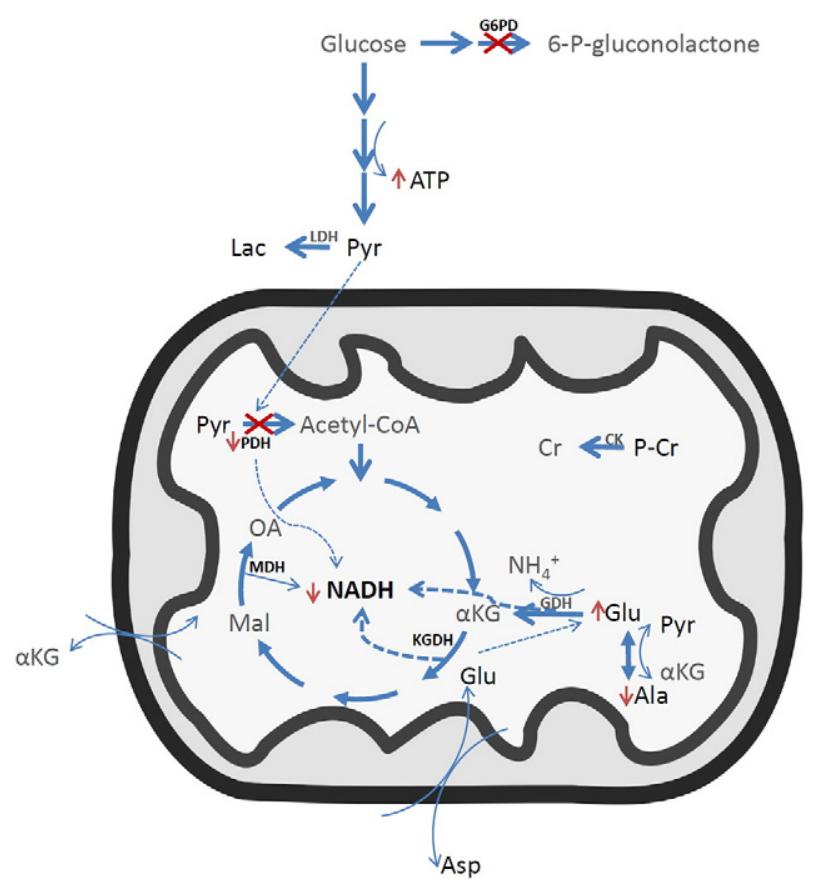

Fig. 8. Bioenergetic dysfunction in HD cybrids, in comparison with CTR cybrids. In HD cybrids glycolysis is stimulated, whereas the pentose phosphate pathway is inhibited. Mitochondrial NADH/NAD levels are decreased due to a decrease in PDH activity and protein levels, although KGDH is stimulated, along with an increase in mitochondrial glutamate levels and a decrease in mitochondrial alanine levels. Phosphocreatine levels contribute to maintain high ATP levels in HD cybrids under glycolysis inhibition conditions. For details see text. Ala- alanine; Asp- aspartate; CK- creatine kinase; $\mathrm{Cr}$ creatine; GDH- glutamate dehydrogenase; Glu- glutamate; KGDH- alpha-ketoglutarate dehydrogenase; $\alpha K G$ - alpha ketoglutarate; Lac- lactate; LDH- lactate dehydrogenase; Mal- malate; $\mathrm{MDH}$ - malate dehydrogenase; OA- oxaloacetate; $\mathrm{P}-\mathrm{Cr}$ - phosphocreatine; PDH- pyruvate dehydrogenase; Pyr- pyruvate. 
Several models have been made available to study mitochondrial dysfunction, being the cybrid technique an interesting approach (King and Attardi, 1989). We recently showed that HD cybrids heighten vulnerability to 3-NP-induced oxidative stress, mitochondrial cytochrome c release and cell death, when compared to CTR cybrids (Ferreira et al., 2010). The analysis of the bioenergetic status of HD cybrids presented in this work reveals that endogenous ATP levels are significantly higher than those present in CTR cybrids. Moreover, that difference was partially refrained upon exposure to 1 or $10 \mathrm{mM}$ 3-NP. The decrease in endogenous ATP levels in HD cybrids exposed to $10 \mathrm{mM}$ 3-NP might be related to the increase in apoptotic cell death observed in these conditions (Ferreira et al., 2010). We also demonstrated that untreated HD cybrids do not exhibit significant modifications in the activity of mitochondrial respiratory chain complexes I-IV, but are endowed with a slight decrease in mitochondrial membrane potential, compared with CTR cybrids (Ferreira et al., 2010). Interestingly, the same HD cybrid lines exhibited increased ATP levels, which were correlated with increased lactate/pyruvate ratio; this ratio decreased upon glycolysis inhibition, revealing increased glycolytic metabolism in HD cybrids. Still, under these conditions, mitochondria supported remaining ATP generation.

Modified glycolytic energy metabolism has been described in HD patients, both in central and in peripheral tissues, as suggested by high levels of lactate in the striatum (Jenkins et al., 1993) and in the cortex (Jenkins et al., 1993; Koroshetz et al., 1997), increased lactate/ pyruvate ratio in the CSF (Koroshetz et al., 1997), but decreased astrocytic glucose metabolism in the striatum with preserved oxygen metabolism in early symptomatic HD patients (Powers et al., 2007b). Moreover, the activity of G6PD, a key enzyme of the pentose phosphate pathway, was decreased in HD cybrids, suggesting that glucose metabolization occurs through the glycolytic pathway. A decrease in total ATP production was further observed in HD cybrids subjected to glycolysis inhibition. Although phosphocreatine levels were shown to be similar between HD and CTR cybrids, glycolysis inhibition significantly increased phosphocreatine consumption in HD cybrids. Phosphocreatine hydrolysis may compensate for the decrease in intracellular ATP levels, since it can function as an alternative energy supply. Nevertheless, unchanged phosphocreatine was observed after inhibition of mitochondrial ATP generation with oligomycin. Previous studies showed low levels of phosphocreatine/ inorganic phosphate ratio in muscle of HD patients compared to control subjects (Lodi et al., 2000) and a delayed recovery of phosphocreatine levels in HD patients in response to exercise (Saft et al., 2005). Peripherally, a significant decrease in phosphocreatine/ inorganic phosphate ratio was found in resting muscle (Koroshetz et al., 1997) of HD patients. Furthermore, pyruvate supplementation (previously shown to stimulate mitochondrial activity in conditions of glycolysis inhibition (e.g. Oliveira et al., 2006; Rego et al., 2001a), did not recover HD cybrids ATP levels. Furthermore, we demonstrated that mitochondrial $\mathrm{NADH} / \mathrm{NAD}_{\mathrm{t}}$ ratio, a parameter that reflects mitochondrial redox status, is decreased in HD cybrids, pointing out the presence of alterations in mitochondrial function upstream oxidative phosphorylation in these cells. The decrease in mitochondrial NADH/NAD $t$ may explain the decrease in mitochondrial membrane potential in HD cybrids, compared to CTR cybrids, despite maintained activity of mitochondrial complexes I-IV (Ferreira et al., 2010). Because these HD cybrid lines are endowed with preserved activity of ETC complexes, the present data suggest a deregulation of mitochondrial function in HD cybrids, upstream the mitochondrial respiratory chain.

At the mitochondrial level, NADH production largely depends on the activity of mitochondrial dehydrogenases, such as PDH, and the TCA enzymes isocitrate dehydrogenase, KGDH and malate dehydrogenase. We observed a large decrease in the activity of PDH in HD cybrids. Nevertheless, the activity of KGDH was increased, suggesting a compensatory mechanism to counterbalance the decrease in NADH production through the PDH. Mitochondrial dehydrogenases are highly regulated enzymes. The activity of these proteins may be affected by availability of substrates and cofactors or by altered expression of the enzymes (Salway, 2006). The activity of mitochondrial dehydrogenases may be also allosterically (e.g. by $\mathrm{Ca}^{2+}$, ATP/ $\mathrm{ADP}, \mathrm{NADH} / \mathrm{NAD}^{+}$) or covalently affected. Covalent modifications that affect enzyme activity include phosphorylation or oxidation. PDH is inhibited by phosphorylation of its E1 alpha subunit and under oxidative stress conditions (Martin et al., 2005). In the present study we show decreased protein levels and increased (although not significantly) Ser phosphorylation of PDH E1 $\alpha$ subunit, which could underlie the decline in PDH activity. Indeed, decreased PDH activity was observed in the caudate and putamen of HD patients (Sorbi et al., 1983). Decreased PDH complex detected in HD patient's caudate nucleus was further correlated with increasing duration of the illness (Butterworth et al., 1985). Moreover, PDH expression was shown to decrease with age in the striatum of R6/2 transgenic mice (Perluigi et al., 2005). Previously, we also showed that HD cybrids present increased ROS production, compared to CTR cybrids (Ferreira et al., 2010); therefore we cannot exclude modifications in PDH activity resulting from enzyme oxidation.

$\mathrm{KGDH}$, another important mitochondrial dehydrogenase, belongs to the same family of PDH complex and shares the same NADH producing subunit, E3 (Gibson et al., 2005). Similarly to PDH, KGDH is affected by oxidative stress, being a mediator between mitochondria and oxidative stress in neurodegeneration (Gibson et al., 2005). Decreased KGDH activity was previously described in Alzheimer's and Parkinson's diseases (Gibson et al., 2000); however, and in contrast with PDH, no changes have been reported in HD. KGDH catalyzed reaction is important for energy production, neurotransmitter metabolism (e.g. glutamate) and metabolic interaction between mitochondria and cytosol. KGDH is also a critical point of TCA regulation, linking mitochondrial bioenergetics to amino acid synthesis and degradation (Gibson et al., 2005). KGDH uses KG produced in the TCA during oxidation of carbohydrates and fatty acids. When the TCA rate is lower than necessary, one way of replenishing TCA intermediaries is through glutamate conversion into KG by mitochondrial glutamate dehydrogenase, which also produces NADH. Since, in our conditions, the TCA does not receive enough input via PDH, KGDH may be a point of entry of TCA intermediaries. In addition, KG may also be produced by transamination of glutamate as part of the malate-aspartate shuttle that transfers reducing equivalents from the cytoplasm to the mitochondria (Gibson et al., 2005). Alteration of glutamate synthesis, metabolism and/or transport in HD cybrids is suggested by the observed increase in mitochondrial glutamate levels, while mitochondrial aspartate levels were unchanged. Although aspartate-glutamate conversions through transamination and glutamine-glutamate deamidation are unlikely to contribute for the increase in mitochondrial glutamate pool, we found a decrease in alanine in mitochondrial subcellular extracts, favoring an increased activity of alanine transaminase. Indeed, mitochondrial glutamate is used by alanine aminotransferase in the mitochondrial matrix to produce pyruvate, by converting KG and alanine into glutamate and pyruvate. In agreement, alanine levels were found to be decreased in the CSF of HD patients, along with decreased pyruvate levels and increased lactate/pyruvate ratio (Koroshetz et al., 1997). A decrease in mitochondrial alanine levels, as an attempt to recover KG levels and thus mitochondrial NADH, may explain the increase in glutamate levels observed under our experimental conditions.

In conclusion, our results show that HD cybrid lines possess inherent bioenergetically dysfunctional mitochondria derived from HD patients' platelets in an otherwise functional nuclear background. Mitochondrial dysfunction persists at the level of PDH, upstream the oxidative phosphorylation, affecting amino acid metabolic fluxes and the cellular bioenergetics through glycolysis stimulation, which assumes a greater importance in promoting ATP production. 


\section{Acknowledgments}

The authors thank the patients and families who generously contributed to this study. We thank Dr. Sandra M. Cardoso and Dr. Sandra Almeida (Center for Neuroscience and Cell Biology, CNC, at University of Coimbra) for helping establishing the HD cybrid lines in our laboratory, to Dr. Isabel Nunes (CNC) for expert technical assistance with cell culture maintenance, and Dr. Russell H. Swerdlow (University of Virginia, Charlottesville, VA, USA) for the gift of rho-zero NT2 cells. This work was supported by Instituto de Investigação Interdisciplinar, Universidade de Coimbra, Portugal (Project III/BIO/49/2005), Gabinete de Apoio à Investigação, Faculdade de Medicina, Universidade de Coimbra (Project STARTER S-09) and Fundação para a Ciência e a Tecnologia, Portugal (Projects POCI/SAU-NEU/57310/2004, PTDC/SAUFCF/66421/2006 and PTDC/SAU-FCF/108056/2008).

\section{References}

Almeida, S., Sarmento-Ribeiro, A.B., Januario, C., Rego, A.C., Oliveira, C.R., 2008. Evidence of apoptosis and mitochondrial abnormalities in peripheral blood cells of Huntington's disease patients. Biochem. Biophys. Res. Commun. 374, 599-603.

Arenas, J., Campos, Y., Ribacoba, R., Martin, M.A., Rubio, J.C., Ablanedo, P., Cabello, A., 1998. Complex I defect in muscle from patients with Huntington's disease. Ann. Neurol. 43, 397-400.

Benchoua, A., Trioulier, Y., Zala, D., Gaillard, M.C., Lefort, N., Dufour, N., Saudou, F., Elalouf, J.M., Hirsch, E., Hantraye, P., Deglon, N., Brouillet, E., 2006. Involvement of mitochondrial complex II defects in neuronal death produced by $\mathrm{N}$-terminus fragment of mutated huntingtin. Mol. Biol. Cell 17, 1652-1663.

Bezprozvanny, I., Hayden, M.R., 2004. Deranged neuronal calcium signaling and Huntington disease. Biochem. Biophys. Res. Commun. 322, 1310-1317.

Bossy-Wetzel, E., Petrilli, A., Knott, A.B., 2008. Mutant huntingtin and mitochondrial dysfunction. Trends Neurosci. 31, 609-616.

Browne, S.E., Bowling, A.C., MacGarvey, U., Baik, M.J., Berger, S.C., Muqit, M.M., Bird, E.D., Beal, M.F., 1997. Oxidative damage and metabolic dysfunction in Huntington's disease: selective vulnerability of the basal ganglia. Ann. Neurol. 41, 646-653.

Budd, S.L., Nicholls, D.G., 1996. A reevaluation of the role of mitochondria in neuronal Ca2+ homeostasis. J. Neurochem. 66, 403-411.

Butterworth, J., Yates, C.M., Reynolds, G.P., 1985. Distribution of phosphate-activated glutaminase, succinic dehydrogenase, pyruvate dehydrogenase and gammaglutamyl transpeptidase in post-mortem brain from Huntington's disease and agonal cases. J. Neurol. Sci. 67, 161-171.

Choo, Y.S., Johnson, G.V., MacDonald, M., Detloff, P.J., Lesort, M., 2004. Mutant huntingtin directly increases susceptibility of mitochondria to the calcium-induced permeability transition and cytochrome c release. Hum. Mol. Genet. 13, 1407-1420.

Choo, Y.S., Mao, Z., Johnson, G.V., Lesort, M., 2005. Increased glutathione levels in cortical and striatal mitochondria of the R6/2 Huntington's disease mouse model. Neurosci. Lett. 386, 63-68.

Ciammola, A., Sassone, J., Alberti, L., Meola, G., Mancinelli, E., Russo, M.A., Squitieri, F., Silani, V., 2006. Increased apoptosis, Huntingtin inclusions and altered differentiation in muscle cell cultures from Huntington's disease subjects. Cell Death Differ. 13, 2068-2078.

DeRosa, G., Swick, R.W., 1975. Metabolic implications of the distribution of the alanine aminotransferase isoenzymes. J. Biol. Chem. 250, 7961-7967.

Ferreira, I.L., Nascimento, M.V., Ribeiro, M., Almeida, S., Cardoso, S.M., Grazina, M., Pratas, J., Santos, M.J., Januario, C., Oliveira, C.R., Rego, A.C., 2010. Mitochondrial-dependent apoptosis in Huntington's disease human cybrids. Exp. Neurol. 222, 243-255.

Gibson, G.E., Park, L.C., Sheu, K.F., Blass, J.P., Calingasan, N.Y., 2000. The alpha-ketoglutarate dehydrogenase complex in neurodegeneration. Neurochem. Int. 36, 97-112.

Gibson, G.E., Blass, J.P., Beal, M.F., Bunik, V., 2005. The alpha-ketoglutarate-dehydrogenase complex: a mediator between mitochondria and oxidative stress in neurodegeneration. Mol. Neurobiol. 31, 43-63.

Gil, J.M., Rego, A.C., 2008. Mechanisms of neurodegeneration in Huntington's disease. Eur. J. Neurosci. 27, 2803-2820.

Gu, M., Gash, M.T., Mann, V.M., Javoy-Agid, F., Cooper, J.M., Schapira, A.H., 1996. Mitochondrial defect in Huntington's disease caudate nucleus. Ann. Neurol. 39, 385-389.

Heinz, F., Weißer, H., 1985. Creatine phosphate. In: Bergmeyer, H.U., Bergmeyer, J., Graßl, M. (Eds.), Methods of Enzymatic Analysis, 3rd edition. VCH-Verlagsgesellschaft, Weinheim, Germany, pp. 507-514.

Jenkins, B.G., Koroshetz, W.J., Beal, M.F., Rosen, B.R., 1993. Evidence for impairment of energy metabolism in vivo in Huntington's disease using localized 1H NMR spectroscopy. Neurology 43, 2689-2695.

Kim, J., Moody, J.P., Edgerly, C.K., Bordiuk, O.L., Cormier, K., Smith, K., Beal, M.F., Ferrante, R.J., 2010. Mitochondrial loss, dysfunction and altered dynamics in Huntington's disease. Hum. Mol. Genet. 19, 3919-3935.

King, M.P., Attardi, G., 1989. Human cells lacking mtDNA: repopulation with exogenous mitochondria by complementation. Science 246, 500-503.
Koroshetz, W.J., Jenkins, B.G., Rosen, B.R., Beal, M.F., 1997. Energy metabolism defects in Huntington's disease and effects of coenzyme Q10. Ann. Neurol. 41, 160-165.

Lodi, R., Schapira, A.H., Manners, D., Styles, P., Wood, N.W., Taylor, D.J., Warner, T.T. 2000. Abnormal in vivo skeletal muscle energy metabolism in Huntington's disease and dentatorubropallidoluysian atrophy. Ann. Neurol. 48, 72-76.

Martin, E., Rosenthal, R.E., Fiskum, G., 2005. Pyruvate dehydrogenase complex: metabolic link to ischemic brain injury and target of oxidative stress. J. Neurosci. Res. 79, 240-247.

Nulton-Persson, A.C., Szweda, L.I., 2001. Modulation of mitochondrial function by hydrogen peroxide. J. Biol. Chem. 276, 23357-23361.

Olah, J., Klivenyi, P., Gardian, G., Vecsei, L., Orosz, F., Kovacs, G.G., Westerhoff, H.V. Ovadi, J., 2008. Increased glucose metabolism and ATP level in brain tissue of Huntington's disease transgenic mice. FEBS J. 275, 4740-4755.

Oliveira, J.M., 2010. Nature and cause of mitochondrial dysfunction in Huntington's disease: focusing on huntingtin and the striatum. J. Neurochem. 114, 1-12.

Oliveira, J.M., Chen, S., Almeida, S., Riley, R., Goncalves, J., Oliveira, C.R., Hayden, M.R. Nicholls, D.G., Ellerby, L.M., Rego, A.C., 2006. Mitochondrial-dependent Ca2+ handling in Huntington's disease striatal cells: effect of histone deacetylase inhibitors. J. Neurosci. 26, 11174-11186.

Panov, A.V., Gutekunst, C.A., Leavitt, B.R., Hayden, M.R., Burke, J.R., Strittmatter, W.J. Greenamyre, J.T., 2002. Early mitochondrial calcium defects in Huntington's disease are a direct effect of polyglutamines. Nat. Neurosci. 5, 731-736.

Parker Jr., W.D., Boyson, S.J., Luder, A.S., Parks, J.K., 1990. Evidence for a defect in NADH: ubiquinone oxidoreductase (complex I) in Huntington's disease. Neurology 40 1231-1234.

Perluigi, M., Poon, H.F., Maragos, W., Pierce, W.M., Klein, J.B., Calabrese, V., Cini, C., De, M.C., Butterfield, D.A., 2005. Proteomic analysis of protein expression and oxidative modification in r6/2 transgenic mice: a model of Huntington disease. Mol. Cell Proteomics. 4, 1849-1861.

Powers, W.J., Haas, R.H., Le, T., Videen, T.O., Hershey, T., McGee-Minnich, L., Perlmutter, J.S., 2007a. Normal platelet mitochondrial complex I activity in Huntington's disease. Neurobiol. Dis. 27, 99-101.

Powers, W.J., Videen, T.O., Markham, J., McGee-Minnich, L., Antenor-Dorsey, J.V., Hershey, T., Perlmutter, J.S., 2007b. Selective defect of in vivo glycolysis in early Huntington's disease striatum. Proc. Natl. Acad. Sci. U. S. A 104, 2945-2949.

Rego, A.C., Vesce, S., Nicholls, D.G., 2001a. The mechanism of mitochondrial membrane potential retention following release of cytochrome c in apoptotic GT1-7 neural cells. Cell Death Differ. 8, 995-1003.

Rego, A.C., Ward, M.W., Nicholls, D.G., 2001b. Mitochondria control ampa/kainate receptor-induced cytoplasmic calcium deregulation in rat cerebellar granule cells. J. Neurosci. 21, 1893-1901.

Rosenstock, T.R., Duarte, A.I., Rego, A.C., 2010. Mitochondrial-associated metabolic changes and neurodegeneration in Huntington's disease - from clinical features to the bench. Curr. Drug Targets 11, 1218-1236.

Saft, C., Zange, J., Andrich, J., Muller, K., Lindenberg, K., Landwehrmeyer, B., Vorgerd, M. Kraus, P.H., Przuntek, H., Schols, L., 2005. Mitochondrial impairment in patients and asymptomatic mutation carriers of Huntington's disease. Mov. Disord. 20, 674-679.

Salway, J.G., 2006. Medical Biochemistry at a Glance 2nd Edition. Wiley-Blackwell.

Sawa, A., Wiegand, G.W., Cooper, J., Margolis, R.L., Sharp, A.H., Lawler Jr., J.F. Greenamyre, J.T., Snyder, S.H., Ross, C.A., 1999. Increased apoptosis of Huntington disease lymphoblasts associated with repeat length-dependent mitochondrial depolarization. Nat. Med. 5, 1194-1198.

Schapira, A.H., 1998. Mitochondrial dysfunction in neurodegenerative disorders. Biochim. Biophys. Acta 1366, 225-233.

Sorbi, S., Bird, E.D., Blass, J.P., 1983. Decreased pyruvate dehydrogenase complex activity in Huntington and Alzheimer brain. Ann. Neurol. 13, 72-78.

Starkov, A.A., Fiskum, G., Chinopoulos, C., Lorenzo, B.J., Browne, S.E., Patel, M.S., Beal, M.F., 2004. Mitochondrial alpha-ketoglutarate dehydrogenase complex generates reactive oxygen species. J. Neurosci. 24, 7779-7788.

Stocchi, V., Cucchiarini, L., Magnani, M., Chiarantini, L., Palma, P., Crescentini, G., 1985. Simultaneous extraction and reverse-phase high-performance liquid chromatographic determination of adenine and pyridine nucleotides in human red blood cells. Anal. Biochem. 146, 118-124.

Swerdlow, R.H., Parks, J.K., Cassarino, D.S., Shilling, A.T., Bennett Jr., J.P., Harrison, M.B., Parker Jr., W.D., 1999. Characterization of cybrid cell lines containing mtDNA from Huntington's disease patients. Biochem. Biophys. Res. Commun. 261, 701-704.

Tabrizi, S.J., Cleeter, M.W., Xuereb, J., Taanman, J.W., Cooper, J.M., Schapira, A.H., 1999 Biochemical abnormalities and excitotoxicity in Huntington's disease brain. Ann. Neurol. 45, 25-32.

Turner, C., Cooper, J.M., Schapira, A.H., 2007. Clinical correlates of mitochondrial function in Huntington's disease muscle. Mov. Disord. 22, 1715-1721.

Umemura, K., Kimura, H., 2005. Determination of oxidized and reduced nicotinamide adenine dinucleotide in cell monolayers using a single extraction procedure and a spectrophotometric assay. Anal. Biochem. 338, 131-135.

Wang, H., Lim, P.J., Karbowski, M., Monteiro, M.J., 2009. Effects of overexpression of huntingtin proteins on mitochondrial integrity. Hum. Mol. Genet. 18, 737-752.

Wick, A.N., Drury, D.R., Nakada, H.I., Wolfe, J.B., 1957. Localization of the primary metabolic block produced by 2-deoxyglucose. J. Biol. Chem. 224, 963-969.

Zhou, Q., Lam, P.Y., Han, D., Cadenas, E., 2008. c-Jun N-terminal kinase regulates mitochondrial bioenergetics by modulating pyruvate dehydrogenase activity in primary cortical neurons. J. Neurochem. 104, 325-335. 